\title{
EVALUACIÓN DE LOS HÁBITOS ALIMENTARIOS DE LOS JÓVENES UNIVERSITARIOS DE LA CIUDAD DE GUAYAQUIL
}

\author{
ASSESMENT OF THE EATING HABITS OF \\ UNIVERSITY STUDENTS IN THE CITY OF GUAYAQUIL
}

\author{
María del Carmen Lapo *1., Ronald Campoverde Aguirre², Daniela Garzón ${ }^{3}$, Priscila Xu³ , Bryan Benítez ${ }^{3}$ \\ 1 Universidad Católica de Santiago de Guayaquil, Facultad de Especialidades Empresariales, Av. Carlos Julio Arosemena km 1.5. maria.lapo@cu.ucsg.edu.ec \\ 2 Escuela Superior Politécnica del Litoral, ESPOL, Facultad de Ciencias Sociales y Humanísticas, Campus Gustavo Galindo Km. 30.5 Vía Perimetral, P.0. Box 09-01-5863, Guayaquil, Ecuador, \\ recampov@espol.edu.ec \\ 3 Universidad Católica de Santiago de Guayaquil, Facultad de Ciencias Económicas y Administrativas, Av. Carlos Julio Arosemena km 1.5
}

El periodo universitario es una etapa de cambios educativos, sociales, familiares, alimentarios $y$ emocionales. Los universitarios se encuentran en un periodo crítico para el desarrollo de estilos de vida que tienen mucha importancia en su futura salud, debido a que la influencia en el comportamiento alimentario de los compañeros, su situación económica y el gusto por cocinar hace que cambien sus hábitos de alimentación.

Objetivo: Distinguir tipologías de los jóvenes universitarios en base a su nivel de satisfacción con la alimentación según los aspectos sociodemográficos, los hábitos y cultura alimentaria.

Método: Se aplicó un cuestionario estructurado a una muestra de 500 estudiantes universitarios de la ciudad de Guayaquil. El instrumento de recogida de información fue tipo escala de Likert. Se consultaron los hábitos de consumo de alimentos dentro y fuera de la universidad, la edad, el peso y estatura aproximada y variables de clasificación sociodemográficas.

Resultados: Mediante análisis factorial se distinguieron cuatro tipologías de estudiantes con diferencias significativas en los puntajes de la escala de satisfacción con la alimentación. Las tipologías difirieron en el número de personas afectadas por preferencia a cocinar, comer fuera de su casa, consumir comida precocinada, planificar su comida, vida social, tradición, conveniencia.

Conclusión: La disponibilidad de los estudiantes para la preparación de su comida incide de forma directa proporcional a su satisfacción con los alimentos. Esto genera que la mayoría de los jóvenes desarrollen un adecuado hábito alimentario, dependiendo de su tiempo para la planificación de sus alimentos y la cocción de este.

Palabras clave: Hábitos alimentarios, jóvenes universitarios, satisfacción con la alimentación.
The university period is a stage of educational, social, family, food and emotional changes. The universitary are in critical period for the development of lifestyles that are very important in their future health, because the influence on the eating behavior on their classmate, their economic situation ans the preference for cooking their own meals change their eating habits.

Aim: Distinguish typologies of universitary students base don their level of satisfaction with the food according to sociodemographic aspects, habits and food culture.

Method: A structured questionnarie was applied to a simple of 500 universitary students from the city of Guayaquil. Thee instrument for collecting information was Likert scale type. The habits of food consumption inside and outsside the place of residence, age, approximate weight and stature, and sociodemographic classification variables were consulted.

Results: Through factor analysis, four types of students were distinguished with signifficant differences in the scores of the satisfaction with food scale. The typologies differed in the number of people affected by prefence to cook, if they eat out of their house, consume precooked food, plan their food, social life, tradition, and convenience.

Conclusion: The availability of students for the preparation of their food affects directly proportional to their satisfaction with food. This means that most of the students develop an adequate food habit, depending on their time for planning their food and cooking it.

Keywords: Food habits, university population, satisfaction with food. 


\section{Introducción}

La alimentación es uno de los factores que más condiciona la salud de los individuos, ejerciendo un papel primordial sobre el desarrollo físico y el crecimiento, la reproducción y el rendimiento físico e intelectual (WHO, 2001). De ahí la importancia que tiene introducir modificaciones en la selección de alimentos de los jóvenes, que permitan seguir unos buenos hábitos alimentarios para promover un estilo de vida saludable.

Según Aranceta (1997) la infancia es una etapa fundamental en la adquisición y desarrollo de hábitos y pautas alimentarias que condicionan el estado nutricional en etapas posteriores de la vida. Si estos hábitos son adecuados, contribuirán a garantizar la salud en la edad adulta (Serra et al., 2002). La dieta de los jóvenes y en especial de los estudiantes universitarios plantea un importante reto, ya que puede suponer cambios importantes en su estilo de vida (Montero, Úbeda, \& García, 2006). El periodo de estudios universitarios suele ser el momento en el cual los estudiantes asumen por primera vez la responsabilidad de su alimentación. (Irazusta, Hoya, \& Díaz, 2007).

Para Birch y Fisher (1998) el aprendizaje de los hábitos alimentarios está condicionado por numerosas influencias procedentes, sobre todo, de la familia (factores sociales, económicos y culturales), del ámbito escolar y a través de la publicidad. Asimismo, Story, Neumark-Sztainer y French (2002) indican que la familia desempeña un papel fundamental en la configuración del patrón alimentario del niño, sin embargo, al alcanzar la adolescencia, el papel de la familia pierde relevancia y el grupo de amigos y las referencias sociales se convierten en condicionantes claves de la dieta del joven adolescente (Cusatis \& Shannon, 1996). Es conocida la vulnerabilidad de este grupo desde el punto de vista nutricional (LópezAzpiazu et al., 2003) y su caracterización por omitir comidas, picar entre horas, abusar de la comida rápida, del alcohol, de las bebidas azucaradas, de las dietas de cafetería y por seguir una alimentación poco diversificada (López, 1999).

Por lo expuesto, se comparte el criterio de Rodríguez et al. (2013) quienes manifiestan, que los estudiantes universitarios son propensos a llevar una mala nutrición, ya que no consumen alimentos entre las comidas centrales, no desayunan, ayunan por largas horas durante el día, siendo particularmente responsables de su propia ingesta de alimentos. La elección de los alimentos, su preparación y las porciones consumidas, influyen directamente en el estado nutricional de los jóvenes universitarios, a pesar de que sus hábitos alimenticios comienzan desde la infancia (Martínez, Veiga, López, Sanz, \& Carbajal, 2005). El nivel de conocimiento en temas relacionados con alimentación y nutrición es un importante determinante de los hábitos de consumo alimentario a nivel individual (Klepp, 1994). Sin embargo, a medida que el individuo adquiere autonomía para decidir comidas y horarios, los factores sociales, culturales y económicos, y las preferencias alimentarias, van a contribuir al establecimiento y al cambio de un nuevo patrón de consumo alimentario de manera importante (Serra et al., 2002).

Sin embargo, con el objetivo de revertir esta problemática, se ha estudiado el tema desde sus diversas vertientes y facetas; una de ellas son, con base a las preferencias alimentarias, las cuales se definen como, la selección de un alimento $u$ otro (Birch, 1999). Los principales factores que influyen en la elección de un alimento son: 1) sociales, tales como, la cultura, la religión, la familia, sus pares, estilos de vida (patrones de comidas, ritmos temporales); 2) culturales los que se derivan del tipo y grado de conocimientos y/o habilidades relativas a los alimentos en general y a la cocina en particular; y 3) económicos que se refieren al costo, los ingresos y la accesibilidad a los diferentes tipos de alimentos, las actitudes, creencias, valores y conocimientos en general (Contreras \& Gracia, 2004). Una de las teorías, erróneamente aceptada, respecto a la etiología de las preferencias alimentarias es que son consideradas, reflejos innatos por la necesidad del cuerpo hacia los nutrientes, la teoría de la "sabiduría del cuerpo", es decir, que nuestro cuerpo pide lo que necesita para nutrirse (Birch, 1999). Otra postura y, quizá la más aceptada, es que las preferencias alimentarias apare $\neg$ cen desde edades tempranas de la vida y se ven influen $\neg$ ciadas por el entorno (Saad et al., 2007). Finalmente, para Uijl, Jager, Graaf, Meiselman y Kremer (2015) la elección y/o preferencias de ciertos alimentos están estrechamente relacionadas con las emociones/recuerdos que produ $\neg$ cen los alimentos y el gusto.

Por otra parte, el conocimiento preciso acerca de la cultura alimentaria es un determinante clave para identificar los factores que influyen en los hábitos alimentarios de los jóvenes universitarios. La cultura alimentaria se entiende como los usos (lujo, placer), tradiciones (religión), costumbres (estatus social, interacción social) y elaboraciones simbólicas de los alimentos (Marín et al., 2004; Vilaplana, 2011) y donde además influye el ciclo productivo de los alimentos del contexto en particular. La cultura alimentaria se puede conocer a través de un diagnóstico situacional, el cual es una etapa básica en la planificación de estrategias o programas (Quintero et al., 2014), ya que aclara problemas regionales y permite vislumbrar posibles soluciones. Del mismo modo, Osorio, Weisstaub, y Castillo (2002) afirman que la cultura alimentaria puede ser definida como un comportamiento habitual relacionado con hábitos alimentarios, selección de alimentos, preparaciones y volúmenes consumidos de estos alimentos, los que influyen directamente en el estado nutricional de los jóvenes universitarios. Mientras que para Cervera, Clapés y Rigolfas (2004) la cultura alimentaria es un fenómeno de naturaleza compleja y de origen multifactorial, que por definición es influenciado por diversos factores entre los cuales se distinguen la sociedad, cultura, religión, situación económica, disponibilidad de alimentos entre otros.

Las conductas alimentarias se adquieren fundamentalmente en el periodo de 6 a 12 años pero pueden verse gravemente alteradas en la etapa de la adolescencia (González \& Merino, 200o). Los jóvenes universitarios, suelen saltarse comidas y desarrollar hábitos alimenticios irregulares, con lo que existe el riesgo de sufrir deficiencias de algunos nutrientes esenciales, desarrollar trastornos de conductas alimentarias o de llegar a sufrir sobrepeso y obesidad. Los hábitos alimentarios inadecuados que se pueden adquirir los estudiantes, en la mayoría de los casos, se mantienen a lo largo de la vida adulta y suponen importantes factores de riesgo para la morbilidad y mortalidad de los adultos (Schneider, 200o; Southon et al., 1994). Debido a esto, algunos estudios 
epidemiológicos muestran que, aunque en ocasiones la población está informada y conoce los conceptos básicos de una dieta saludable, estos conocimientos no se traducen en consumos reales de alimentos que formen parte de una dieta equilibrada. No se ponen en la práctica los conceptos aprendidos (López, 2002).

Hoy en día, se ha incrementado la preocupación internacional por los problemas de nutrición en la adolescencia y la juventud, sobre todo en relación a la prevalencia de sobrepeso, obesidad y patologías asociadas, así como también por sus consecuencias negativas en los ámbitos psicológico y social (Schnettler et al., 2013). La modernización de la sociedad ha supuestouna serie de cambios sociológicos y/o culturales que afectan inevitablemente a los hábitos y preferencias alimentarias. Se dedica menos tiempo a la compra de alimentos y elaboración de las comidas y, a cambio, se prefieren los alimentos procesados (Durá \& Castroviejo, 2011).

En este sentido, la presente investigación tiene por objetivo determinar y evaluar los factores que influyen en los hábitos alimentarios de los jóvenes universitarios de la ciudad de Guayaquil.

\section{Metodología}

El tipo de investigación contempla un diseño no experimental transversal correlacional, con un enfoque cuantitativo. La muestra corresponde a jóvenes universitarios de instituciones de educación superior pública y privada de la ciudad de Guayaquil, que en total representantas 500 estudiantes, mediante el uso del muestreo estratificado, quienes se encuentran en el rango de 17 a 30 años. El tamaño maestral fue determinado con un margen de error de 0.05 , supuesto de mayor incertidumbre $p=q=0,5 \mathrm{y}$ un nivel de confianza del $95 \%$.

Para evaluar los hábitos alimenticios se aplicó un cuestionario tipo escala de Likert, denominada "Encuesta para la evaluación de los hábitos alimentarios de los jóvenes universitarios de la ciudad de Guayaquil" del estudio de Schnettler et al. (2013). El juicio de experto permitió contrastar cada uno de los ítems con la teoría en relación a los hábitos alimentarios de los estudiantes. La encuesta tiene por objetivo medir los hábitos alimentarios de los sujetos y se compone de tres ámbitos, el primer ámbito abarca los aspectos sociodemográficos del estudiante (edad, género, tipo de Universidad, peso, estatura). Se consultó cuanto de efectivo destino el estudiante para su alimentación diaria. Adicional a esto, se consultó la satisfacción con la alimentación mediante una escala tipo Likert de cuatro niveles (1: extremadamente satisfecho, 4: insatisfecho). El segundo ámbito compuesto por catorce ítems que exploran la auto percepción de los estudiantes acerca de sus hábitos alimentarios, los encuestados debieron responder su grado de acuerdo o desacuerdo con las afirmaciones mediante una escala tipo Likert de 5 niveles (1: siempre, 5 nunca). Finalmente, el tercer ámbito sobre la cultura alimentaria del estudiante consta de cinco ítems, (Gusto por cocinar, vida social, tradición, conveniencia, planificación), de igual forma este ámbito fue medida mediante una escala tipo Likert de 5 niveles (1: siempre, 5 nunca)

Los estudiantes fueron contactados en sus clases habituales de la universidad. El cuestionario fue auto complementado por los estudiantes en una sola sesión sin interrupciones y todos durante la misma semana en junio de 2019.

\section{Técnicas a utilizar}

El análisis de los resultados fue realizado con el programa SPSS 22.0 en español para Windows. Se aplicó estadística descriptiva a los factores sociodemográficas (género, edad, tipo de Universidad, peso y estatura). Las variables cuantitativas se han expresado en términos de media y desviación estándar y las variables cualitativas se expresan en términos de porcentajes. La extracción de factores de las escalas se realizó con análisis factorial de componentes principales, considerando autovalores mayores que 1. La consistencia interna de las escalas fue calculada usando el coeficiente de Cronbach.

Por último, se estimó una regresión logística binaria para determinar los factores que inciden en la satisfacción alimentaria de los jóvenes, a través de la siguiente especificación:

$Y_{i}=f\left(D_{1 i}, X_{1 i}, D_{2 i}, X_{2 i}, X_{3 i}, X_{4 i}, D_{3 i}, D_{4 i}, D_{4 i}, D_{6 i}, D_{7 i}, D_{8 i, U i}\right.$

Dónde:

Yi =Satisfacción con la alimentación

$\mathrm{D} 1 \mathrm{i}=$ Genero

$\mathrm{X} 1 \mathrm{i}=\mathrm{Edad}$

D2i = Tipo de Universidad

$\mathrm{X} 2 \mathrm{i}=$ Estatura

$\mathrm{X} 3 \mathrm{i}=$ Peso

$\mathrm{X} 4 \mathrm{i}=$ Gasto en alimentación diaria

D3i = Hábitos alimentarios

D4i $=$ Gusto por cocinar

D5i $=$ Vida Social

D6i $=$ Tradición

D7i $=$ Conveniencia

D8i = Planificación

$\mathrm{Ui}=$ Errores $\mathrm{y} / \mathrm{o}$ variables omitidas

\section{Resultados}

La muestra estuvo constituida por un mayor número de participantes de género femenino (57.8\%), y de universidad pública $(79.6 \%)$. La edad promedio de la muestrafue21.16años (desv. estand.=2.88). La estatura y peso promedio fue de 5.94 (desv. estand.=26.35) y 63.64 (desv. estand.=14.30) respectivamente. Finamente, el gasto medio en alimentos fue de 5.28 dólares (desv. estand.=3.51).

Tabla 1 Variables sociodemográficas de la muestra

\begin{tabular}{lll}
\hline \multicolumn{1}{c}{ Variable } & $\mathrm{n}$ & $\%$ \\
\hline Género & & \\
\hline Femenino & 289 & 57.8 \\
Masculino & 211 & 42.2 \\
\hline Típo de Universidad & & \\
\hline Pública & 398 & 79.6 \\
Privada & 102 & 20.4 \\
\hline
\end{tabular}


Se obtuvo los índices de fiabilidad para las dos variables de estudio. La Tabla 2 muestra los resultados de Cronbach todos los coeficientes superaron el valor de 0.70 , lo que refleja que las escalas son confiables.

Tabla 2 Coeficiente de Alfa de Cronbach

\begin{tabular}{lcc}
\hline \multicolumn{1}{c}{ Variables } & Alfa de Cronbach & Items \\
\hline Hábitos alimentarios &, 767 & 14 \\
Cultura alimentaria &, 702 & 5 \\
\hline
\end{tabular}

Antes de la reducción de dimensiones se obtuvo el KMO con un valor de .772 indica que los datos son aptos para la aplicación del análisis factorial. La Tabla 3 muestra las cargas en los 14 ítems agrupados ahora en 4 factores que explican en conjunto el $60.22 \%$ de la varianza. El primer factor agrupa los ítems del 1 al 4 , que reflejan el perfil de jóvenes que preparan sus alimentos, éste componente explicó la mayor parte de la varianza $19.46 \%$. El segundo constructo contiene los reactivos del 5 al 8, que indican el perfil de jóvenes que prefieren comer fuera, el cual presenta una varianza del $14.51 \%$. La tercera dimensión abarca los ítems 9 al 11, que responden al perfil de jóvenes que optan por comida precocinada, con una varianza del $14.31 \%$. Por último el cuarto componente comprende los reactivos del 12 al 14, que muestran el perfil de jóvenes que planifican su alimentación, explicando la menor parte de la varianza $11.93 \%$.

Tabla 3 Factores de los hábitos de consumo

\begin{tabular}{|c|c|c|c|c|}
\hline & \multicolumn{4}{|c|}{ Componente } \\
\hline & 1 & 2 & 3 & 4 \\
\hline Le gusta cocinar & ,830 & , O73 & ,025 & 124 \\
\hline Es un excelente cocinero/a & ,846 & ,017 & ,116 & ,106 \\
\hline $\begin{array}{l}\text { Ser reconocido por sus habilidades de cocina, es muy importante } \\
\text { para su auto-estima }\end{array}$ & ,704 & , 033 & ,182 & ,157 \\
\hline Busca formas nuevas de preparar comidas & ,823 & , 045 &,- 018 & ,101 \\
\hline Salir a comer fuera es parte de sus hábitos alimentarios regulares & ,008 &, 554 & ,292 &,- 146 \\
\hline Le gusta compartir con amigos disfrutando de una comida en la Universidad &,- 016 &, 775 &,- 016 & , o66 \\
\hline Las recetas conocidas son las mejores & ,174 & ,716 & ,042 & ,146 \\
\hline Sólo compra alimentos que le son familiares & ,005 & ,687 & ,066 & ,065 \\
\hline Usa bastantes alimentos precocinados en su cocina & , 040 & ,114 & ,833 & , 002 \\
\hline $\begin{array}{l}\text { Usa bastantes mezclas preparadas, por ejemplo masas para hornear } \\
\text { y sopas instantáneas }\end{array}$ & 131 &,- 025 & ,835 & ,123 \\
\hline Los alimentos congelados son una parte importante de su despensa & , O71 & ,171 & ,612 & ,164 \\
\hline Lo que van a cenar se planifica generalmente durante la hora de almuerzo. & ,110 & , o89 & ,103 &, 799 \\
\hline Planifica lo que van a comer, con un par de días de anticipación, & ,131 &,- 141 & ,290 &, 747 \\
\hline Le agrada mantener sus hábitos alimentarios & ,250 & ,247 &,- 068 &, 565 \\
\hline Varianza explicada & 19,463 & 14,515 & 14,316 & 11,933 \\
\hline Varianza acumulada & 19,463 & 33,979 & 48,295 & 60,228 \\
\hline Alfa de Cronbach & 833 & ,653 & ,701 & ,603 \\
\hline Prueba de Barlett & 1832,016 & & &, 772 \\
\hline
\end{tabular}

Finalmente, se estimó tres modelos agregando los factores sociodemográficos, de hábitos alimentarios, y cultura alimentaria. El primer modelo se incorporó los hábitos alimentarios, se obtuvo significativa solo la variable jóvenes que planifican su comida con un $\beta=.37$ y R2= .034. Para llegar a tener resultados más viables, se añadió al modelo anterior las variables de cultura alimentaria, donde el coeficiente gusto por cocinar fue el único que resulto estadísticamente significativo $\beta=4.86$, este modelo presento un $\mathrm{R} 2=.075$. Adicional a esto para tener un $\mathrm{R}_{2}$ más confiable, se estimó un tercer modelo con todos los factores incorporados, resultando significativos los estimadores: jóvenes que preparan sus alimentos $\beta=3.13$; gusto por cocinar $\beta=4.98$; planificación $\beta=3.29$ $y$ edad $\beta=3.67$, concluyendo con un $R_{2}=.122$. Cabe recalcar que la variable gusto por cocinar es la que mejor explica al modelo de la satisfacción alimentaria de los jóvenes universitarios. 
Tabla 4 Modelo Logit

\begin{tabular}{|c|c|c|c|c|c|c|}
\hline \multirow[b]{2}{*}{ Variables } & \multicolumn{2}{|c|}{ Modelo 1} & \multicolumn{2}{|c|}{ Modelo 2 } & \multicolumn{2}{|c|}{ Modelo 3} \\
\hline & Beta & Wald & Beta & Wald & Beta & Wald \\
\hline Jóvenes que preparan sus alimentos & , ০ & ,০o &, 39 & 1,99 &, 52 & $3,13^{*}$ \\
\hline Jóvenes que comen fuera & ,25 & 1,98 & ,28 & 1,81 & ,29 & 1,78 \\
\hline Jóvenes que consume alimentos precocinados &,- 17 & ,78 &,- 16 & ,65 &,- 11 & ,28 \\
\hline Jóvenes que planifican su comida &, 37 & $3,52^{*}$ &, 36 & 2,50 &, 37 & 2,53 \\
\hline Gusto por cocinar & & &,- 53 & $4,86^{*}$ &,- 55 & $4,98^{* *}$ \\
\hline Vida Social & & &,- 15 & ,39 &,- 24 & ,92 \\
\hline Tradición & & &,- 05 & ,05 &,- 11 & ,20 \\
\hline Conveniencia & & & ,10 & ,19 & ,15 & ,44 \\
\hline Planificación & & & ,29 & 2,14 & ,37 & $3,29^{*}$ \\
\hline Género & & & & &,- 55 & 1,37 \\
\hline Edad & & & & &, 13 & $3,67^{*}$ \\
\hline Universidad & & & & & ,14 & , 08 \\
\hline Estatura & & & & &,- 06 & , 01 \\
\hline Peso & & & & & , 02 & 1,84 \\
\hline Gasto & & & & & , 01 & ,05 \\
\hline Constante & & & & & $-5,93$ & $8,62^{* * *}$ \\
\hline R2 de Nagelkerke & .034 & & .075 & & .122 & \\
\hline
\end{tabular}

${ }^{*} \mathrm{p}<0.05,{ }^{* *} \mathrm{p}<0.01$

\section{Discusiones y Conclusiones}

Con la ayuda del análisis de componentes principales fue posible agrupar 14 ítems en 4 factores, tales como el perfil de estudiantes que preparan sus alimentos, el perfil de estudiantes que prefieren comer fuera, el perfil de estudiantes que optan por comida precocinada y el perfil de estudiantes que planifican su alimentación. Factores que al agregar los aspectos sociodemográficos y la cultura alimentaria permitieron evaluar y determinar los hábitos alimentarios de los estudiantes universitarios de la ciudad de Guayaquil.

Los resultados del presente estudio afirman que los estudiantes universitarios de la ciudad de Guayaquil encuentran una mayor satisfacción a la hora de consumir alimentos preparados y planificados por ellos mismos, además, dichos resultados revelan que la edad es un aspecto directamente influyente a la hora de determinar el nivel de responsabilidad alimentaria, es decir, mientras más adulto sea el estudiante, mayor será su preferencia por preparar y planificar su alimentación. Pese a que los estudiantes encuentran una mayor satisfacción al preparar su propio alimento, prefieren no hacerlo, esto se debe a que el gusto por la cocina en jóvenes es realmente bajo. Por lo tanto, un alto nivel de responsabilidad alimentaria se traduce a una correcta adquisición de hábitos alimentarios a través del tiempo.

\section{Referencias}

Aranceta, J.(1997). Nutriciónenelniñoyadolescente. DiálogosenPediatría 9. Meneghello J (ed). Santiago de Chile, Mediterráneo, p.146-144.
Birch, L. (1999). Development of food preferences. Annual Reviews, p. 41-62. Birch, L., \& Fisher, J. (1998). Development of eating behaviors among children and adolescents. Pediatrics, p. 593-594. Cervera, R., Clapés, J., \& Rigolfas, R. (2004). Alimentación y dietoterapia. Colombia: 4a Edición. Editorial McGraw - Hill Interamericana. Contreras, J., \& Gracia, M. (2004). Preferencias y consumos alimentarios: entre el placer, la conveniencia y la salud. Observatorio de la Alimentación. y la salud. Observatorio de la Alimentación.
Cusatis, D., \& Shannon, B. (1996). Influences on adolescent eating behavior. J Adolesc Health, p. 27-34. Durá Travé, T., \& Castroviejo Gandarias, A. (Abril de 2011). Nutrición Hospitalaria. Obtenido de Adherencia a la dieta mediterránea en la población universitaria. González, E., \& Merino, B. (200o). Guía de Nutrición Saludable y Prevención de los Trastornos Alimentarios. . Madrid: MISC-MEC. Irazusta, A., Hoya, I., \& Díaz, E. (2007). Alimentos de A. estudiantes \& universitarios. Osasunaz. Klepp, K. (1994). welve year follow-up of a school-based health education programme. The Oslo Youth Study. Eur Public Health , p. 195-200. Koninklijke, G., \& Thieme, N. (1965). Aspectos sociales y económicos del planeamientodela educación. Organizacióndelas NacionesUnidas paralaEducación, laCienciayla CulturaplacedeFontenoy. Nimega.

López Nomdedeu, C. (1999). Influencia de la estructura social y familiar en el desarrollo de los hábitos alimentarios. Tratado de Nutrición, pp. 1355-1365. López Nomdedeu, C. (2002). Educación nutricional de niños/ as $\mathrm{y}$ adolescentes. Alimentación Infantil y Juvenil.

López-Azpiazu, I., Sánchez-Villegas, A., Johansson, L., Petkeviciene, J., Prattala, R., \& Martínez-González, M. (2003). Disparities in food habits in Europe: systematic review of educational and occupational differences in the intake of fat. Nutr Diet, p. 349-364.

Marín, G., Álvarez, M., \& Rosique, J. (2004). Cultura alimentaria en el municipio de Acandí. Boletín de Antropología. Universidad de Antioquía, Vol. 18( Núm. 35.).

Martínez, C., Veiga, P., López, A., Sanz, C., \& Carbajal, A. (Junio de 2005). Nutrición Hospitalaria. Obtenido de Evaluación del esstado nutricional de un grupo de estudiantes universitarios mediante parámetros dietéticos y de composición corpros

Montero, A., Úbeda, N., \& García, A. (2006). Evaluación de los hábitos alimentarios de una población de estudiantes universitarios en relaciónconsusconocimientosnutricionales. NutrHosp, p. 446-473. Osorio, J., Weisstaub, G., \& Castillo, C. (2002) Desarrollo
de la conducta alimentaria en la infancia sus alteraciones a Rev chil Nutr, $3,280-285$. Pérez-Rodrigo, C. Ribas, Lev Chir Nutr, \& 3, 280-28 opiniones sobre temas relacionados con alimentación

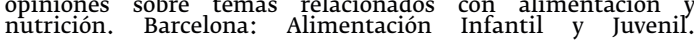


Quintero, J., Cardona, H., Rodríguez, Y., Rolong, J., \& Salas, M. (2014). Diagnóstico situacional de las condiciones importantes y necesarias para la implementación de Redes Integradas de Servicios de Salud (RISS) basadas en Atención Primaria en Salud (APS) en el departamento de Huila. Entornos(Núm. 27). Rodríguez, F., Palma, X., Romo, Á., Escobar, D., Aragú, B., Espinoza, L., . . Gálvez, J. (2013). Nutrición Hospitalaria. Obtenido de Hábitos alimentarios, actividad física y nivel socioeconómico en estudiantes universitarios de chile.

Saad, C., Ibáñez, E., León, C., Colmenares, C., Vega, N., \& Díaz, Y. (2007). Cambios de los hábitos alimentarios de los estudiantes de enfermería de la Universidad El Bosque durante su proceso de formaciónacadémica. Rev. Colombianadeenfermería, 3, 1909-1621.

Schneider, D. (2000). International trends in adolescent nutrition. . Social Science \& Medicin, 51, 955-967. Schnettler, B., Denegrí, M., Miranda, H., Sepúlveda, J., Orellana, L., \& Grunert, K. (Noviembre de 2013). Nutrición Hospitalaria. Obtenido de Hábitos alimentarios y bienestar subjetivo en estudiantes universitarios del sur de Chile.

Serra, L., Ribas, L., García, R., Pérez-Rodrigo, C., Peña, L., \& Aranceta, J. (2002). Hábitos alimentarios y consumo de alimentos en la población infantil y juvenil española (1998200o): variables socioeconómicas y geográficas. Barcelona: Estudio Enkid. Serra L. Aranceta J (eds.). Masson, 14-28.
Southon, S., Wright, J., Finglas, P., Bailey, A., Loughridge, J., \& Walker, A. (1994). Dietary intake and micronutrient status of adolescents: effect of vitamin and trace element supplementation on indices of status and performance of verbal and nonverbal on indices of status and performance of verbal and nonverbal
intelligence. British Journal of Nutrition, 71, 897-987. intelligence. British Journal of Nutrition, 71, 897-987.
Story, M., Neumark-Sztainer, D., \& French, S. (2002). Individual and and environmental $\begin{gathered}\text { French, S. } \\ \text { influences }\end{gathered}$ adolescent eating behaviors. J Am Diet Asso, p. 40-51. Uijl, L., Jager, G., Graaf, C., Meiselman, H. , \& Kremer, S. (2015). Emotion, olfaction, and age: A comparison of self-reported food-evoked emotion profiles of younger adults, oldernormosmic adults, and older hyposmic adults. Food Quality and Preference, 48, 199-209. Vilaplana, M. (2011). Cultura alimentaria. Del fast food al slow food. Offarm., Vol. 30 (Núm. 6). WHO, R. o. ( 2001). Nutrition and Food Security Programme. 